Gyanesh K. Gupta

William W. Schultz

Ellen M. Arruda

Xiaoyong $\mathrm{Lu}$

\section{Nonisothermal model of glass fiber drawing stability}

Received: 25 July 1996

Accepted: 23 September 1996

Dedicated to the memory

of Professor Tasos C. Papanastasiou

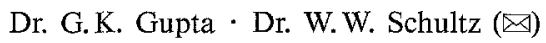

Dr. E. M. Arruda - X. Lu

Department of Mechanical Engineering

and Applied Mechanics

University of Michigan

Ann Arbor, Michigan 48109, USA

\begin{abstract}
Draw resonance is caused by a constant speed winder that leads to non-constant axial forces (Schultz, 1984). The well studied isothermal Newtonian fiber drawing predicts very modest critical draw ratios (around 20, much less than the typical production draw ratios for glass fibers of $10^{3}-10^{5}$ ). The nonisothermal fiber drawing model presented here shows that cooling along the spin line strongly stabilizes the process. However, we show that the conclusion of Shah and Pearson $(1972 a, b)$ that nonisothermal Newtonian fiber spin-
\end{abstract}

ning is unconditionally stable is based on non-converged numerical results. The choice of viscosity-temperature correlation function has a strong influence in determining the stability of the process. While viscoelasticity generally has an adverse effect on the stability, low viscoelasticity in the presence of extensional thinning helps to slightly improve the maximum critical draw ratio.

Key words Glass - Fiber drawing - draw resonance nonisothermal

- White-Metzner model

\section{Introduction}

The properties of glass fibers and those of the same material in bulk form are usually quite different. During glass fiber drawing, the material is rapidly stretched as it experiences severe thermal gradients such that the fibers solidify into a non-equilibrium state. Hence, it is reasonable to conclude that viscoelastic effects are important at some location on the spin line. The fiber properties and the process stability depend strongly on process parameters such as melt temperature (temperature at the oriffice), heat transfer to the environment (radiation and convection), draw ratio and winder speed.

For high-speed operation, an undesirable phenomenon called draw resonance limits the production rates for a stable process. Draw resonance is characterized by periodic oscillations in tension in the drawn filament. Draw resonance is caused by a constant speed winder that leads to nonconstant axial forces (Schultz, 1984).
There have been significant experimental and theoretical efforts in the past three decades to study the effects of various parameters on the draw resonance instability. Most previous efforts concentrate on studying Newtonian or non-Newtonian isothermal fiber drawing. Recently, Gupta and Schultz (1996) have analyzed steady, nonisothermal Newtonian fiber drawing. We shall here consider the thermal effects for unsteady drawing of glass fibers. Since glass fibers have extensive applications from telecommunications to composite materials, the study of nonisothermal draw resonance in glass fibers has practical importance.

Petrie and Denn (1976), and Denn (1980) provide extensive literature reviews on draw resonance in fiber drawing. When viscoelastic, inertia, surface tension, gravity and thermal effects are neglected, the predicted critical draw ratio (ratio of the average fiber speed at the winder to that at the orifice, $E=w_{w} / w_{i}$ ) is approximately 20.21 (Petrie and Denn, 1976). This is much less than the typical production draw ratios for glass fibers of $10^{3}-10^{5}$. 
This isothermal result is now well established and serves as a benchmark for testing numerical methods for studying draw resonance. The goal of this study is to carefully determine how nonisothermal and viscoelastic effects inhibit draw resonance.

Shah and Pearson (1972a) correlate the critical draw ratio for nonisothermal Newtonian fiber drawing with a parameter $S$ representing convective heat transfer. They report the process is unconditionally stable beyond a critical $S$. They also find increasing convection does not ensure greater stability. Shah and Pearson (1972b) find that inertia has a similar effect. In all these studies, they have further simplified the basic state governing equations using an exponential temperature dependence on $z$ that is not affected by the kinematics of the problem, rather than solving the coupled steady state governing equations. The findings of Chang et al. (1981) on the stability of continuous isothermal drawing of Newtonian fibers incorporating the effects of inertia, gravity and surface tension agree qualitatively with Shah and Pearson (1972a). Pearson and Shah (1974) extend the nonisothermal analysis to power-law fluids. They find that the "relation between critical extension rate and power-law index proves to be monotonic, while the effect of cooling seems to be far less effective with 'strain-rate-thinning' fluids than with Newtonian fluids." The strain-rate thinning in their modeling occurs for extensional or shear flow. Chang et al. (1982) find that a weak temperature-viscosity dependence enhances the stabilization process. Vasiljev and Naumchic (1990) conclude that for an optical glass fiber-drawing process there is an optimum temperature condition for maximum stability. Mhaskar and Shah (1977) show that heat transfer increases the stability for Newtonian fiber drawing. They also show that surface tension has destabilizing effects. The experiments of Demay and Agassant (1985) for isothermal drawing of polymer fibers are in good agreement with the stability curves computed using a nonisothermal Newtonian or a viscoelastic Maxwell model. The analyses of Cao (1991, 1993) for isothermal draw resonance in power-law fluids agree with Pearson and Shah (1974). Myers (1989) considers draw resonance in fibers drawn from glass preforms where the radiative exchange is the dominant form of heat transfer. Like Shah and Pearson (1972a), he finds unconditional stability for sufficiently high heat transfer. Kase (1974) also finds that cooling stabilizes the process, although he does not determine critical draw ratios.

Most previous studies of viscoelastic fiber drawing assume an isothermal process and focus on the portion of the fiber away from the orifice where the flow is taken to be one-dimensional. This means that the axial velocity and pressure are independent of the radial coordinate or that the axial velocity has become rectilinear. Denn et al. (1975), Gupta et al. (1986), Sridhar et al. (1986), Papanastasiou et al. (1987), Phan-Thien (1987), Schultz (1987), and Nguyen et al. (1990), have addressed viscoelastic iso- thermal fiber drawing using either Maxwell or Oldroyd-B viscoelastic models. However, nonisothermal effects on viscoelastic fiber drawing have received little attention. Recently, Wang and Forest (1994) have considered steady nonisothermal fiber spinning of a Maxwell liquid using an Arrhenius type viscosity-temperature dependence. In the present study, we show an Arrhenius viscosity-temperature dependence does not fit the experimental data well for glass for the entire range of temperatures in spinning.

There are fewer studies where viscoelastic effects are analyzed in stabilizing the process. Fisher and Denn (1976) study draw resonance of isothermal melt spinning of a White-Metzner viscoelastic fluid. They find the existence of lower and upper critical draw ratios. Beris and Liu (1988), Liu and Beris (1988), and Anturkar and Co (1988) also find the existence of two critical draw ratios for an isothermal viscoelastic fluid. They find that the fiber drawing process is stable at low and high draw ratios. However, the lower critical value is found to be unaffected by the magnitude of the viscoelastic effects. Furthermore, Schultz (1987) questions the validity of onedimensional modeling for higher extension ratios where viscoelasticity affects the process stability.

Fisher and Denn (1977) find that both viscoelasticity and cooling are stabilizing, and the system becomes absolutely stable to infinitesimal disturbances for sufficiently large viscoelastic effects. However, large viscoelasticity leads to an unattainable region at larger draw ratios. This region corresponds to infinite drawing forces. Hence, there is little gain in stable draw ratios. Pearson et al. (1976) also find that freezing of the fiber in the spinline improves stability and that elasticity appears to destabilize the process.

In glass fiber drawing, the flow field is predominantly one-dimensional except within one diameter of the orifice for typical parameter ranges. Glass is nearly a Newtonian fluid when hot, and a Hookean solid when cool. In fiber drawing, the material goes from fluid to solid state without a sudden change in phase (without devitrification). Our preliminary experiments show that fiber properties depend strongly on the thermal-strain history. The measurements of Sammler et al. (1996) also show typical viscoelastic effects near glass transition temperatures. Hence, we seek a viscoelastic model that is able to describe the material response in the entire drawing domain and for various post-processing steps, such as tempering. A realistic model would consider an integral model as in Chen and Papanastasiou (1990). Instead, we use a simple differential upper-convected Maxwell model with strain rate and temperature-dependent properties (the modified Maxwell model is also widely known as the White-Metzner model) that is commonly used for fiber drawing.

Since the fiber length is several orders of magnitude larger than the orifice diameter, the one-dimensional flow analysis should be appropriate except when viscoelastic 
effects dominate, especially near the orifice. A lubrication analysis (Schultz, 1987) systematically derives the onedimensional equations without an a priori one-dimensional assumption for small viscoelastic effects. However, most viscoelastic one-dimensional models to date include viscoelastic effects at leading order. In this study, for simplification, we also include viscoelastic effects at leading order. While we recognize that viscoelasticity and heat transfer can lead to invalidation of the one-dimensional model, their combination can have a beneficial effect without loss of validity. That is, where the fiber is hot, the glass is Newtonian and most of the deformation will occur there. By the time viscoelastic effects become important, the deformation is small, so locally the weakly elastic criteria of Schultz (1987) holds. Therefore, the more simple model appears to be valid except near the unattainable region that typically occurs in upper-convected Maxwell models (Fisher and Denn, 1976). Our preliminary studies testing the validity of the one-dimensional model have confirmed this.

The one-dimensional governing equations (conservation of mass, momentum and energy) are coupled and a closed-form basic state solution is not available. Thus, an eigenvalue analysis requires a numerical simulation of the basic state solution as well as the linearized stability system of equations that become ordinary differential equations in the axial coordinate. In many previous nonisothermal studies (Shah and Pearson, 1972a; Pearson et al., 1976; Mhaskar and Shah, 1977; Fisher and Denn, 1977) the basic state solution is further simplified by assuming that the energy equation is decoupled from the momentum equations. We solve the coupled basic state governing equations numerically using B splines. We present a one-dimensional model that explores the influence of viscoelasticity and heat transfer in stabilizing the process. The present model shows that while heat transfer stabilizes the fiber drawing operation, viscoelasticity generally has an adverse effect on the process stability. However, in the presence of extensional thinning, low viscoelasticity slightly improves the maximum critical draw ratio. No study has yet addressed numerical convergence of the critical draw ratio for nonisothermal drawing. We address these issues and establish a criterion for determining spurious eigenvalues that can lead to erroneous conclusions.

In the next section, we summarize the derivation of the dimensionless unsteady governing equations following the procedure of Gupta and Schultz (1996) and provide the basic state solution in the third section. The linearized perturbation analysis is carried out in the fourth section. In the fifth section we discuss the validity of our numerical scheme and nonisothermal results are discussed in the sixth section. Concluding remarks and future work are discussed in the last section.

\section{Governing equations}

We analyze the glass fiber drawing process based on the upper convected Maxwell fluid model with temperaturedependent and shear-rate dependent viscosity and relaxation functions (other properties such as density and thermal conductivity are considered constant). This incorporates the essential characteristics of the material in this simulation. We ignore the effects of inertia, gravity, surface tension and viscous dissipation.

We consider a slender, axisymmetric fiber emerging from an orifice of radius $R_{i}$ (subscript $i$ refers to the quantities at the orifice) into a passive gaseous environment. At a distance $L$ the fiber is "wound up" after being stretched at a given average axial velocity $w_{w}$ (subscript $w$ refers to the quantities at the winder). A cylindrical coordinate system is shown in Fig. 1.

We choose a simple differential constitutive equation for the fluid and consider an upper convected Maxwell model given as

$$
\begin{aligned}
& \mathbf{T}=-p \mathbf{I}+\mathbf{S}, \\
& \mathbf{S}+\frac{\eta(\theta, I I)}{G}\left(\frac{\mathscr{D} \mathbf{S}}{\mathscr{D} t}-a(\mathbf{S} \cdot \mathbf{D}+\mathbf{D} \cdot \mathbf{S})\right)=2 \eta(\theta, I I) \mathbf{D},
\end{aligned}
$$

where $\mathbf{T}$ is the Cauchy stress tensor, $\mathbf{I}$ is the identity tensor, $p$ is the pressure, $\mathbf{S}$ is the extra Cauchy stress tensor,

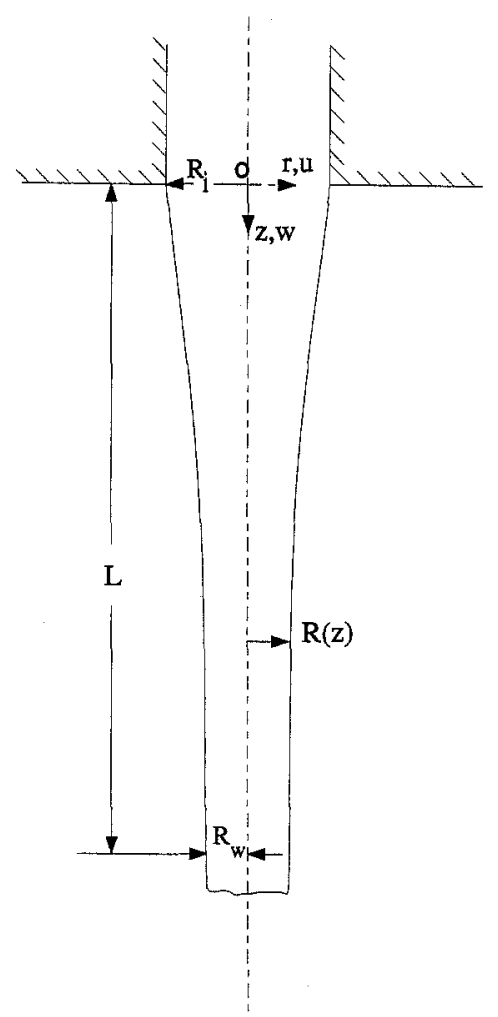

Fig. 1 Schematic of fiber drawing process 
$\eta$ is the viscosity function, $a$ is a rate parameter (here, we usually consider only $a=1$ for the upper-convected model), $G$ is the modulus, $\theta$ is the absolute temperature, $\mathbf{D}$ is the shear-rate tensor, $I I$ is the second invariant of $\mathbf{D}$, and $\mathscr{D} / \mathscr{D} t$ is the Jaumann derivative. The variation in the modulus for glass is typically found to be less than $20 \%$ (Varshneya, 1995) for a wide range of temperature. Hence, the relaxation time $\eta / G$ is assumed to have the same temperature and strain-rate dependence as the viscosity in accordance with the thermo-rheological simplicity assumption ( $G$ is constant), except where noted.

One widely used temperature-viscosity correlation is a simple exponential (Gupta and Schultz, 1996; Glicksman, 1968) fitting a modest temperature range, typically for moderately high temperatures where most deformation takes place. However, the correlation does not capture the temperature dependence of viscosity of E-glass over a large range of temperatures. An Arrhenius function in Fig. 2 is valid for all temperatures (other than absolute zero), but does not fit E4 glass data well. Figure 2 also shows a William-Lendel-Ferry (WLF) temperature-viscosity correlation (Bird, 1987), a commonly used improvement on the Arrhenius function which has three parameters to fit the data. However, this function with parameters chosen for the higher temperature range has a singularity at a finite temperature (approximately $270^{\circ} \mathrm{C}$ ) much higher than the ambient temperature. A simulation based on such a model is not valid for a nonisothermal study all the way to the winder. A Walther temperatureviscosity correlation (O'Donnell and Zakarian, 1984) has only two parameters but fits the experimental data (Larsen, 1980) for a wide range of temperature. We choose this function as it does not have a singularity for the temperature interval $\theta_{\infty}<\theta<\theta_{i}$, where $\theta_{i}$ is the average orifice absolute temperature, and $\theta_{\infty}$ is the absolute ambient temperature.

We also account for extensional thickening (or thinning) behavior. A Carreau power-law dependence on the second invariant of the strain-rate tensor II (Bird, 1987) is considered for the extensional-rate-viscosity dependence. For one-dimensional extensional flow $I I$ is proportional to $d w / d z$. Based on the above criteria the following viscosity function is chosen in the present study:

$$
\eta\left(\theta, \frac{d w}{d z}\right)=\frac{\eta_{0}}{\left(1+\delta \frac{d w}{d z}\right)^{q}} \exp \left[\exp \left(v_{0}-v_{1} \ln \theta\right)\right]
$$

where $v_{0}$ and $v_{1}$ are viscosity-temperature coefficients, $q$ is the power-law index, and $\delta$ and $\eta_{0}$ are positive constants. Here, we set $\delta=1$. This correlation is not singular except at absolute zero temperature or when $d w / d z<0$.

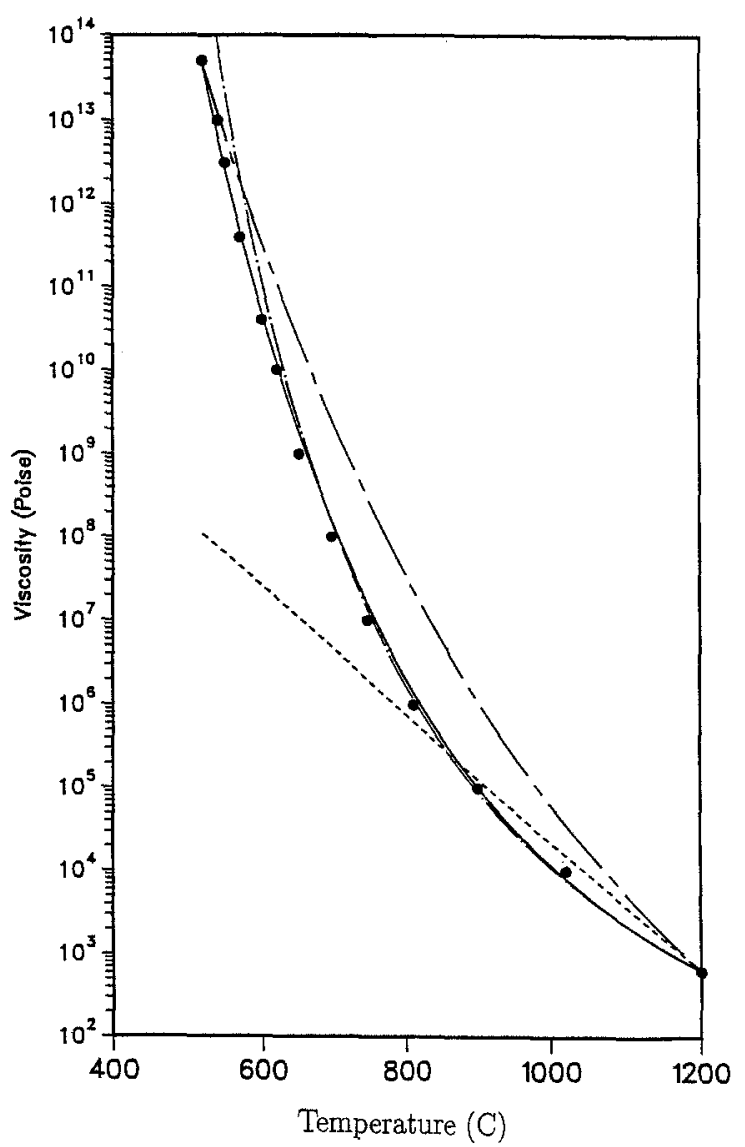

Fig. 2 Viscosity-temperature correlations for E4 Glass (here, $\theta$ is in degrees Kelvin). Experiment (Larsen, 1980); Walther correlation: $\exp (\exp (20.5682-2.5641 \ln \theta)) ;---\operatorname{Sim}-$ ple exponential: $1.3293 \times 10^{14} \exp (-0.01769 \theta)$; - - - WLF correlation: $10^{-1.7+(4177 / \theta-474)}$; $\ldots$ Arrhenius correlation: $\exp (-22.8217+43113 / \theta)$

We consider the heat-transfer coefficient $h$ (which may include radiation effects) to be a function of the local kinematic parameters (Kase and Matsuo, 1965),

$$
h=\frac{k_{\infty}}{2 R} C\left(\frac{2 \rho_{\infty} w R}{\eta_{\infty}}\right)^{m}
$$

where $k_{\infty}, \rho_{\infty}$ and $\eta_{\infty}$ are the thermal conductivity, density and the viscosity of the ambient air at room temperature, respectively. The coefficients $C$ and $m$ are determined from the experimental data. To combine the effects of radiation in the heat-transfer coefficient, the constant $C$ and the power-law coefficient are modified from those in Kase and Matsuo (1965).

Following Schultz and Davis (1982), we apply lubrication scaling to the governing equations and develop all expansions in powers of $\varepsilon=R_{i} / L$. We define the following dimensionless variables: 


$$
\begin{array}{r}
r^{*}=\frac{r}{R_{i}}, \quad z^{*}=\frac{z}{L}, \quad u^{*}=\frac{u}{\varepsilon w_{i}}, \quad w^{*}=\frac{w}{w_{i}}, \\
\eta^{*}=\frac{\eta}{\eta_{i}}, \quad \mathbf{T}^{*}=\frac{\mathbf{T}}{w_{i} \eta_{i} / L}, \quad \theta^{*}=\frac{\theta-\theta_{\infty}}{\theta_{i}-\theta_{\infty}},
\end{array}
$$

where the superscript $*$ refers to the nondimensional quantities. The following dimensionless parameters are defined:

$$
\mathrm{Pe}=\frac{\rho w_{i} R_{i} C_{p}}{k \varepsilon}, \quad \mathrm{Bi}=\frac{1}{\varepsilon^{2}} \frac{h R_{i}}{k} .
$$

Here, $\rho, C_{p}$ and $k$ are the density, specific heat and thermal conductivity of glass, respectively. Though these glass properties are all functions of temperature, for simplification they are considered constant here. By requiring the Biot number to be scaled in the above manner with $\mathrm{Bi}=O(1)$, the leading-order solution for $\theta$ depends only on the axial coordinate consistent with the one-dimensional assumption.

The unsteady one-dimensional dimensionless governing equations are obtained from the leading-order equations following the procedure of Gupta and Schultz (1996). The superscript * is dropped for notational convenience, henceforth all variables are considered as nondimensional. Conservation of mass and axial momentum give:

$$
\begin{aligned}
& \frac{\partial}{\partial t}\left(R^{2}\right)+\frac{\partial}{\partial z}\left(w R^{2}\right)=0 . \\
& \frac{\partial}{\partial z}\left[R^{2}\left(T_{z z}-T_{r r}\right)\right]=0 .
\end{aligned}
$$

The unsteady energy equation includes advection, axial conduction and heat-transfer to the ambient due to convection. Viscous dissipation is neglected as the Brinkman * number is small. Heat loss due to radiation is often small compared to convective heat losses and hence it is lumped in the convective heat-transfer coefficient for simplicity. This heat transfer model is different from previous analyses in that it includes thermal conduction, and hence has an extra term in the unsteady energy equation:

$$
\operatorname{Pe}\left(\frac{\partial \theta}{\partial t}+w \frac{\partial \theta}{\partial z}\right)=\frac{1}{R^{2}} \frac{\partial}{\partial z}\left(R^{2} \frac{\partial \theta}{\partial z}\right)-\frac{2 \mathrm{Bi}}{R} \theta .
$$

The Peclet number Pe is an inverse dimensionless thermal conductivity and the Biot number $\mathrm{Bi}$ is a dimensionless heat transfer coefficient. Thermal conductivity must be present in the model to formally derive the one-dimensional equations (Gupta and Schultz, 1996). The model requires that the Peclet and Biot numbers be $O$ (1). Since these numbers in our simulation can become quite large, there is some concern about two-dimensional effects (Bechtel et al., 1992). We will not consider these complications here.

The unsteady dimensionless conservation laws for mass, momentum and energy along with the constitutive equation form the governing set of equations for the stability analysis. A linearized perturbation analysis following the steady-state solution determines the temporal stability.

\section{The basic state solution}

The steady basic state solution (designated by overbar) for the fiber radius $\bar{R}$ is obtained from the mass conservation equation as

$$
\bar{R}=\bar{w}^{-1 / 2} \text {. }
$$

The axial momentum equation gives a uniform (independent of $z$ ) axial force $\bar{F}$, as

$$
\frac{\bar{S}_{z z}-\bar{S}_{r r}}{\bar{w}}=\bar{F},
$$

and the axial temperature distribution $\bar{\theta}(z)$ is obtained by the energy conservation equation as

$$
\frac{d^{2} \bar{\theta}}{d z^{2}}-\left(\frac{1}{\bar{w}} \frac{d \bar{w}}{d z}+\operatorname{Pe} \bar{w}\right) \frac{d \bar{\theta}}{d z}-2 \sqrt{\bar{w}} \operatorname{Bi} \bar{\theta}=0 .
$$

The constitutive equation provides a first-order differential system for stress terms given by

$$
\begin{aligned}
& \frac{\bar{\eta}}{G} \bar{w} \frac{d \bar{S}_{r r}}{d z}+\left(1+a \frac{\bar{\eta}}{G} \frac{d \bar{w}}{d z}\right) \bar{S}_{r r}+\bar{\eta} \frac{d \bar{w}}{d z}=0, \\
& \frac{\bar{\eta}}{G} \bar{w} \frac{d \bar{S}_{z z}}{d z}+\left(1-2 a \frac{\bar{\eta}}{G} \frac{d \bar{w}}{d z}\right) \bar{S}_{z z}-2 \bar{\eta} \frac{d \bar{w}}{d z}=0 .
\end{aligned}
$$

The above system of equations is then solved with the following boundary conditions (Gupta and Schultz, 1996):

$$
\begin{aligned}
& \bar{w}=1, \quad \bar{R}=1, \quad \text { and } \bar{\theta}=1 \quad \text { at } \quad z=0, \\
& \bar{w}=e^{\alpha}=\ln E, \quad \text { and } \frac{d^{2} \bar{\theta}}{d z^{2}}=0 \quad \text { at } \quad z=1,
\end{aligned}
$$

where $\alpha$, known as the Hencky strain, is the critical stability parameter. An upstream stress boundary condi- 
tion completes the problem statement. We choose to impose a boundary condition at the orifice based on the Newtonian limit consistent with the one-dimensional model (Schultz, 1987). In addition, the glass is hot at the orifice, so it is not very elastic there. Then

$$
\operatorname{tr}(\bar{S})=0 \quad \text { at } \quad z=0 .
$$

\section{The linearized stability analysis}

We seek a solution of the governing equation in the form

$$
\phi=\bar{\phi}(z)\left[1+\phi_{p}(z, t)\right],
$$

where $\phi$ represents the quantities $w, R, \theta$, and $S$, the overbar denotes the basic state variables; and the subscript $p$ denotes the perturbed quantity. The proportional disturbances of this form were first used by Pearson and Matovich (1969) to simplify the stability analysis. Substituting (18) in the unsteady dimensionless equations of conservation of mass, momentum and energy and collecting terms linear in the perturbation quantities gives the governing equations for the stability analysis.

The perturbation quantities $\phi_{p}$ are solved in normalmode form

$$
\phi_{p}=\hat{\phi}(z) e^{\sigma t},
$$

where $\hat{\phi}$ is the eigenfunction representing $\hat{w}, \hat{R}, \hat{\theta}, \hat{S}_{r r}$, and $\hat{S}_{z z}$, and the eigenvalue is $\sigma=\sigma_{r}+i \sigma_{i}$, where $i=\sqrt{-1}$. If $\sigma_{r}$ is positive, then the disturbances will grow and the flow is unstable. Substituting (19) for the perturbation variables in the stability equations and eliminating nonlinear terms in perturbation quantities gives the following linear, coupled, homogeneous differential equations:

$$
\begin{aligned}
& \bar{w} \frac{d \hat{w}}{d z}+2 \bar{w} \frac{d \hat{R}}{d z}=-2 \sigma \hat{R}, \\
& \frac{d}{d z}\left[2 \hat{R} \bar{F}+\frac{\left(\bar{S}_{z z} \hat{S}_{z z}-\bar{S}_{r r} \hat{S}_{r r}\right)}{\bar{w}}\right]=0,
\end{aligned}
$$

$$
\bar{\eta}\left(\frac{d \bar{w}}{d z}+\frac{\bar{w}}{G} \frac{d \bar{S}_{r r}}{d z}+\frac{a \bar{S}_{r r}}{G} \frac{d \bar{w}}{d z}\right)\left[1-\frac{q \delta \frac{d \bar{w}}{d z}}{\left(1+\delta \frac{d \bar{w}}{d z}\right)}\right] \hat{w}
$$

$$
\begin{aligned}
& +\bar{w} \bar{\eta}\left[\left(1+\frac{a \bar{S}_{r r}}{G}\right)\right. \\
& \left.-\left(\frac{d \bar{w}}{d z}+\frac{\bar{w}}{G} \frac{d \bar{S}_{r r}}{d z}+\frac{a \bar{S}_{r r}}{G} \frac{d \bar{w}}{d z}\right) \frac{q \delta \frac{d \bar{w}}{d z}}{\left(1+\delta \frac{d \bar{w}}{d z}\right)}\right] \frac{d \hat{w}}{d z} \\
& +\left[\frac{-\bar{\eta} \bar{\theta}\left(\theta_{i}-\theta_{\infty}\right) v_{1}}{\left\{\theta_{\infty}+\left(\theta_{i}-\theta_{\infty}\right) \bar{\theta}\right\}}\right.
\end{aligned}
$$

$$
\begin{aligned}
& \times \exp \left[v_{0}-v_{1} \ln \left(\theta_{\infty}+\bar{\theta}\left(\theta_{i}-\theta_{\infty}\right)\right)\right] \\
& \left.\times\left(\frac{d \bar{w}}{d z}+\frac{\bar{w}}{G} \frac{d \bar{S}_{r r}}{d z}+\frac{a \bar{S}_{r r}}{G} \frac{d \bar{w}}{d z}\right)\right] \hat{\theta} \\
& +\left[\bar{\eta}\left(\frac{\bar{w}}{G} \frac{d \bar{S}_{r r}}{d z}+\frac{a \bar{S}_{r r}}{G} \frac{d \bar{w}}{d z}\right)+\bar{S}_{r r}\right] \hat{S}_{r r} \\
& +\frac{\bar{\eta} \bar{w} \bar{S}_{r r}}{G} \frac{d \hat{S}_{r r}}{d z}=-\sigma \frac{\bar{\eta} \bar{S}_{r r}}{G} \widehat{S}_{r r},
\end{aligned}
$$

$\bar{\eta}\left(2 \frac{d \bar{w}}{d z}-\frac{\bar{w}}{G} \frac{d \bar{S}_{z z}}{d z}+\frac{2 a \bar{S}_{z z}}{G} \frac{d \bar{w}}{d z}\right)\left[1-\frac{q \delta \frac{d \bar{w}}{d z}}{\left(1+\delta \frac{d \bar{w}}{d z}\right)}\right] \hat{w}$

$$
+\bar{w} \bar{\eta}\left[2\left(1+\frac{a \bar{S}_{z z}}{G}\right)\right.
$$$$
-\left(2 \frac{d \bar{w}}{d z}-\frac{\bar{w}}{G} \frac{d \bar{S}_{z z}}{d z}+\frac{2 a \bar{S}_{z z}}{G} \frac{d \bar{w}}{d z}\right)
$$$$
\left.\frac{q \delta \frac{d \bar{w}}{d z}}{\left(1+\delta \frac{d \bar{w}}{d z}\right)}\right] \frac{d \hat{w}}{d z}+\left[\frac{-\bar{\eta} \bar{\theta}\left(\theta_{i}-\theta_{\infty}\right) v_{1}}{\left\{\theta_{\infty}+\left(\theta_{i}-\theta_{\infty}\right) \bar{\theta}\right\}^{2}}\right.
$$

$$
\times \exp \left[v_{0}-v_{1} \ln \left(\theta_{\infty}+\bar{\theta}\left(\theta_{i}-\theta_{\infty}\right)\right)\right]
$$$$
\left.\times\left(2 \frac{d \bar{w}}{d z}-\frac{\bar{w}}{G} \frac{d \bar{S}_{z z}}{d z}+\frac{2 a \bar{S}_{z z}}{G} \frac{d \bar{w}}{d z}\right)\right] \hat{\theta}
$$$$
+\left[-\bar{S}_{z z}+\bar{\eta}\left(\frac{2 a \bar{S}_{z z}}{G} \frac{d \bar{w}}{d z}-\frac{\bar{w}}{G} \frac{d \bar{S}_{z z}}{d z}\right)\right] \hat{S}_{z z}
$$ 


$$
\begin{aligned}
& -\frac{\bar{\eta} \bar{w} \bar{S}_{z z}}{G} \frac{d \hat{S}_{z z}}{d z}=\sigma \frac{\bar{\eta} \bar{S}_{z z}}{G} \hat{S}_{z z}, \\
& \frac{d^{2} \hat{\theta}}{d z^{2}}-\left[\left(\frac{1}{\bar{w}} \frac{d \bar{w}}{d z}+\bar{w} \mathrm{Pe}\right)-2 \bar{\theta}^{-1} \frac{d \bar{\theta}}{d z}\right] \frac{d \hat{\theta}}{d z} \\
& +2 \bar{\theta}^{-1} \frac{d \bar{\theta}}{d z} \frac{d \hat{R}}{d z}+2 \operatorname{Bi} \sqrt{\bar{w}} \hat{R}-\bar{w} \operatorname{Pe} \bar{\theta}^{-1} \frac{d \bar{\theta}}{d z} \hat{w}= \\
& -\sigma \operatorname{Pe} \hat{\theta} \text {. }
\end{aligned}
$$

The above system of equations along with homogeneous versions of boundary conditions $(15-17)$ form a differential eigenvalue system for $\sigma$.

\section{Numerical approach and validation}

We divide the domain $[0,1]$ into $N$ intervals and use orthogonal B splines (de Boor, 1978) to seek an eigensolution of the form

$$
\widehat{\phi}=\sum \varphi_{j} B_{j}(z)
$$

where $\varphi_{j}$ are unknown coefficients. Equation (25) is then substituted into the governing differential eigenfunction Eqs. (20-24) and homogeneous boundary conditions. The resulting residuals are then minimized using the Galerkin method using a weight function $B_{i}(z)$. This procedure converts the differential eigensystem into an algebraic eigensystem whose eigenfunctions are the unknown coefficients $\varphi$. The eigenspectra are then obtained using standard EISPACK routines. We also solve the same differential eigensystem using central finite differences. This parallel approach quantifies the errors associated with various approximations, and helps to optimize the number of intervals $N$ for accurate analysis.

We begin by comparing the results using $B$ splines and central finite differences with the well established Newtonian isothermal result. Table 1 compares the growth rates $\min \left(\sigma_{r}\right)$ obtained numerically with those obtained earlier (Schultz and Davis, 1984). The $\min \left(\sigma_{r}\right)$ from B splines is accurate to five significant digits when the size of the eigensystem $N$ is 100 for an extension ratio between

Table 1 Isothermal Newtonian fiber drawing, $\alpha=5.05$

\begin{tabular}{llll}
\hline Method & $N$ & $\min \sigma_{r}$ & $\mid$ Error $\mid$ \\
\hline Exp. integral & & +4.80571 & 0 \\
B splines & 25 & +4.80866 & 0.00295 \\
& 50 & +4.80586 & 0.00015 \\
Finite difference & 100 & +4.80574 & 0.00003 \\
& 100 & +4.75294 & 0.05277 \\
& 200 & +4.79262 & 0.01309 \\
\hline
\end{tabular}

the isothermal and nonisothermal critical limits, and for which the result from the exponential integral is available. The finite difference approach gives only one digit accuracy at $N=100$ and two digits for $N=200$ consistent with quadratic convergence. A further increase in $N$ to give a comparable result would be computationally expensive. Hence, we pursue only the Galerkin approach for further comparison and analysis.

\section{Nonisothermal computational results}

\section{Generalized Newtonian model}

Figure 3 presents marginal stability curves for glass-fiber drawing simulations for the simple exponential viscosity temperature correlation. We draw marginal stability curves as a function of the Biot number at the winder $\operatorname{Bi}(z=1)$ that is changed by varying the constant $C$ in the heat-transfer relation (4). We do not observe unconditional stability once the effective solidification of the fiber is achieved as reported by Shah and Pearson (1972a,b), Pearson and Shah (1974), Mhaskar and Shah (1977), and Myers (1989). This reflects that after the fiber is sufficiently solidified, there is no further deformation and hence no further increase in the critical draw ratio. Although the fiber is essentially solidified, the process can be unstable - essentially moving the downstream boundary conditions at the winder to that at the effective solidification location. We attribute the conclusions of Shah and Pearson (1972a,b), Pearson et al. (1974),

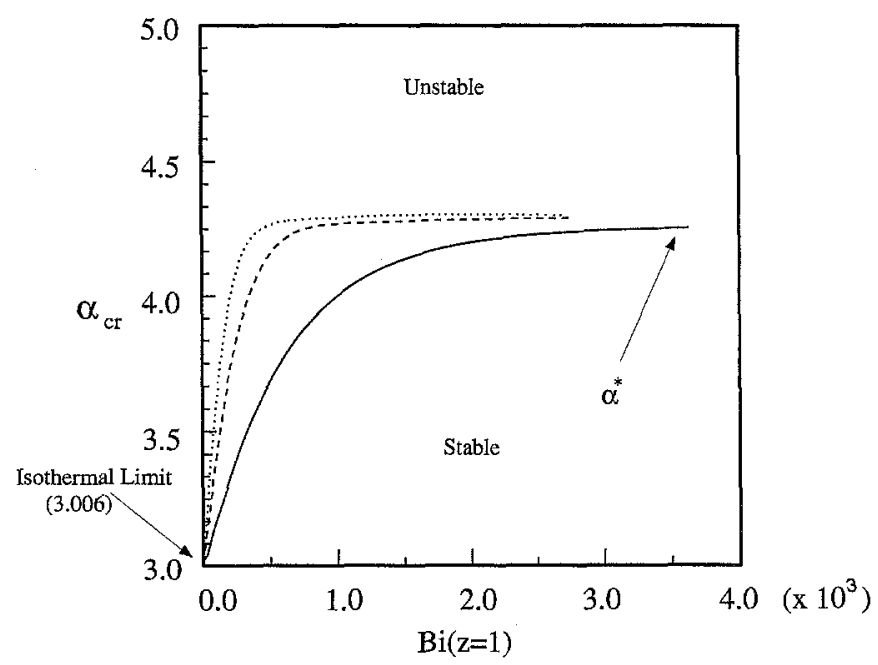

Fig. 3 Marginal stability curves for nonisothermal Newtonian fiber drawing for simple exponential viscosity-temperature correlations as a function of the Biot number at the winder, $P e=474$, $\mathrm{m}=0.13, q=0$ (here, $\theta$ is in degrees Kelvin). $1.3293 \times 10^{14} \exp (-0.00769 \theta) ;---1.3293 \times 10^{14} \exp (-0.01769$ $\theta) ; \ldots \ldots 1.3293 \times 10^{14} \exp (-0.02769 \theta)$ 
Mhaskar and Shah (1977), to the following two factors: they have further simplified the governing equations using an exponential temperature dependence on $z$ that is not affected by the kinematics of the problem rather than solving a coupled steady state governing equation, and their finite-difference results are under-resolved for the higher extension ratios. Myers (1989) solved the energy equation in a more conventional manner, but he did not give details about the numerical convergence nor the viscosity relationship other than the statement, "In this paper it will be assumed that the glass instantaneously freezes (and unfreezes during heating) when it reaches $T_{s}$ [the softening point]."

We solve the coupled governing equations numerically using B splines. We have also shown that the eigenvalues obtained by the finite-difference method are not very accurate as compared to the pseudo-spectral Galerkin method using B splines even for the isothermal case. A very fine mesh is required to obtain convergent eigenvalues for large draw ratios. In essence, the mesh has to be refined nearly exponentially as the Hencky strain $\alpha$ increases linearly. Previous analyses did not address convergence as examined in Table 2. This tabulation is important for two reasons: to avoid spurious roots, and to obtain convergent (mesh independent) eigenvalues.

When calculating eigenvalues, unstable modes often occur with large growth rates that become unbounded as $N$ increases. These "spurious modes" arise due to discretization of the continuous problem and due to round-off and truncation errors. The Galerkin method is one of the most accurate methods for studying the eigenvalue problem arising in stability analysis (Zebib, 1987), yet this method can have spurious modes. These modes are usually easily identifiable as they are large in magnitude and vary considerably with $N$. The critical eigenvalue often also has the smallest modulus $M=\sqrt{\sigma_{r}^{2}+\sigma_{i}^{2}}$. Hence, to locate this critical value we look for eigenvalues with the smallest real part as well as the smallest modulus.

Table 2 shows that the process becomes unstable as the draw ratio is increased. However, at low $N$, the process appears to restabilize with a further increase in the draw ratio. When we consider a finer mesh the temporal root

Table 2 Nonisothermal Newtonian fiber drawing: $\min \left(\sigma_{r}\right)$ for simple exponential model (Gupta and Schultz, 1996; Glicksman, 1968), $\mathrm{Pe}=474, C=4.0, m=0.33$

\begin{tabular}{llll}
\hline$\alpha$ & $N=20$ & $N=60$ & $N=100$ \\
\hline 1.0 & -7.17370 & -7.15855 & -7.15843 \\
2.0 & -6.94378 & -6.86397 & -6.86232 \\
3.0 & -4.37698 & -4.45617 & -4.45380 \\
4.0 & -0.66533 & -0.64305 & -0.64777 \\
5.0 & +1.76126 & +4.36039 & +4.31626 \\
6.0 & +0.93108 & +10.8215 & +10.7341 \\
7.0 & -6.02248 & +18.8188 & +19.0790 \\
8.0 & -24.5310 & +27.7654 & +29.7668 \\
\hline
\end{tabular}

value changes not only its value but also its sign, deciding the stability of the process. As the draw ratio is increased, a finer mesh is required to obtain a convergent eigenvalue, as can be observed from Table 2. Hence, for the large draw ratios studied in the present work, we confirm the eigenvalues obtained using $N=100$ by further refining the mesh size when required. A further refinement in the mesh does improve the estimate of the eigenvalue to obtain mesh independence. However, the computational time increases quadratically with an increase in the mesh size. Most of the results reported here are based on a mesh size $N=100$.

Figure 3 shows that different viscosity-temperature dependencies affect the stability. However, for significantly large heat loss (sufficient to make the glass temperature at the winder close to ambient temperature) all values of the simple exponential coefficient approach the same asymptotic value, that we will here call $\alpha^{*}$. There is a strong correlation between the heat transfer represented by the Biot number at the winder $\operatorname{Bi}(z=1)$ and the glass temperature at the winder $\theta(z=1)$. Figure 4 shows the data of Fig. 3 replotted against $\theta(z=1)$ that shows $\alpha_{\mathrm{cr}}$ has reached its asymptotic value $\alpha *$ for typical temperatures at the winder. Hereafter, we plot the stability curve as a function of $\theta(z=1)$. Figures 3 or 4 show that the simple exponential temperature viscosity relationship cannot explain the high stable production draw ratios for the different cases considered.

Figure 5 shows marginal stability results for the different viscosity-temperature correlations shown in Fig. 2. We observe that the asymptotic predictions from the WLF and Walther viscosity-temperature correlations are nearly

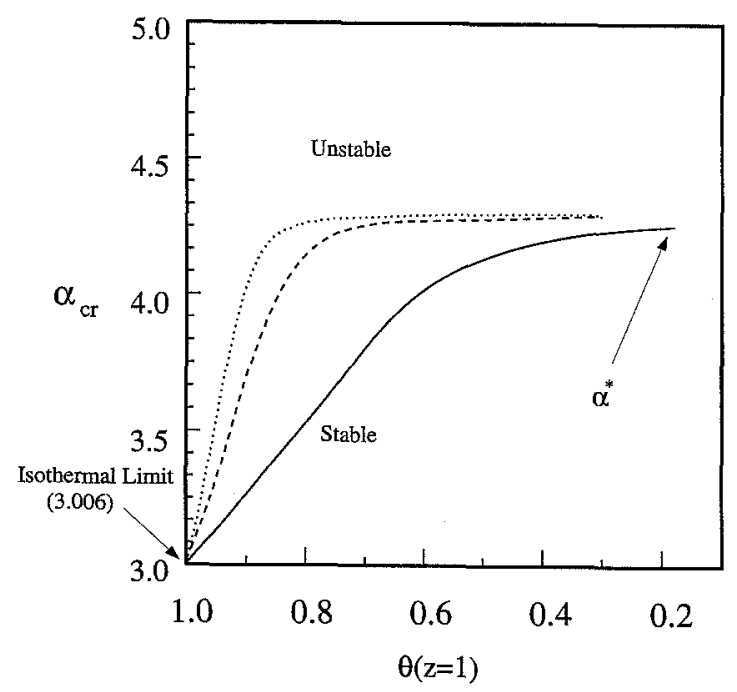

Fig. 4 Marginal stability curve for nonisothermal Newtonian fiber drawing for simple exponential viscosity-temperature correlation, $\mathrm{Pe}=474, \mathrm{~m}=0.13, q=0$ (here, $\theta$ is in degrees Kelvin). $1.3293 \times 10^{14} \exp (-0.00769 \theta) ;-\ldots-1.3293$ $\times 10^{14} \exp (-0.01769 \theta) ; \ldots \ldots .1 .3293 \times 10^{14} \exp (-0.02769 \theta)$ 


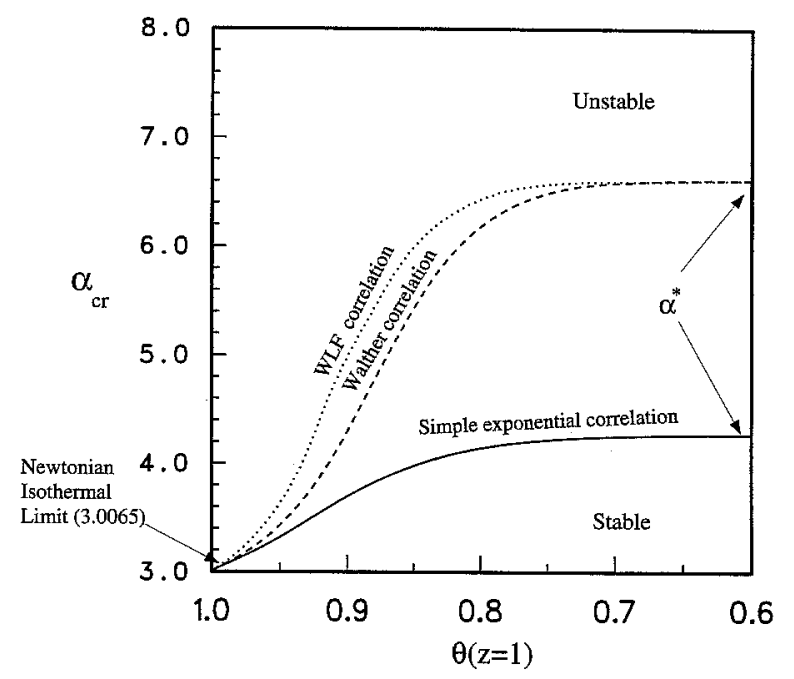

Fig. 5 Marginal stability curve for nonisothermal Newtonian fiber drawing for different viscosity-temperature correlations, $\mathrm{Pe}=474$, $\mathrm{m}=0.13, q=0$

the same as long as the temperature of the winder is sufficiently low but above the singular temperature of the WLF function. However, they both are much different from the simple exponential correlation. Hence, heat transfer has a stabilizing influence on the process stability, but it is strongly affected by the shape of the viscositytemperature correlation, not just by how rapidly the glass solidifies. While the simple exponential correlation predicts a modest increase in critical draw-ratio, the Walther correlation predicts stable draw ratios close to those found in production. Although the WLF correlation has similar stability characteristics as the Walther correlation, it is singular in the range $0<\theta<1$. The Walther correlation is not singular except at absolute zero temperature. The Newtonian nonisothermal maximum critical draw ratio for the Walther function is about 1000 . While significantly higher than the maximum critical draw ratio of about 70 for the simple exponential correlation, it is still less than the commonly observed draw ratios in industrial fiber production. Hence, we focus on the effect of different non-Newtonian parameters on the marginal stability using only the Walther viscosity-temperature correlation.

Figure 6 shows the effect of the power-law index in combination with heat transfer. As in the case of isothermal drawing, we observe that extensional thickening $(q<0)$ increases the maximum critical draw ratio. The critical draw ratio is predicted to approach 10000 for the nonisothermal generalized Newtonian fiber drawing with a power-law index $q=-0.5$. Extensional thinning $(q>0)$ on the other hand has a destabilizing influence on the process stability. Unlike the viscosity-temperature relationship, measurements of extension thickening are not available, but they are unlikely to be far from zero. We plot $\alpha *$ for a range of power-law indices in Fig. 7.

Figure 8 shows the effect of Peclet number on the critical draw ratio. All previous stability studies neglect axial conduction assuming an infinite Peclet number. Based on the value of glass conductivity and parameters in our analysis, the Peclet number is 474 . Figure 8 shows that including axial conduction reduces stability, but not significantly as the maximum critical draw ratio for $\mathrm{Pe}=474$ is close to its asymptotic value as $\mathrm{Pe} \rightarrow \infty$.

Therefore, except for questionably low values of $q$, the stability of the generalized Newtonian model is still much less than that commonly observed in production. Since

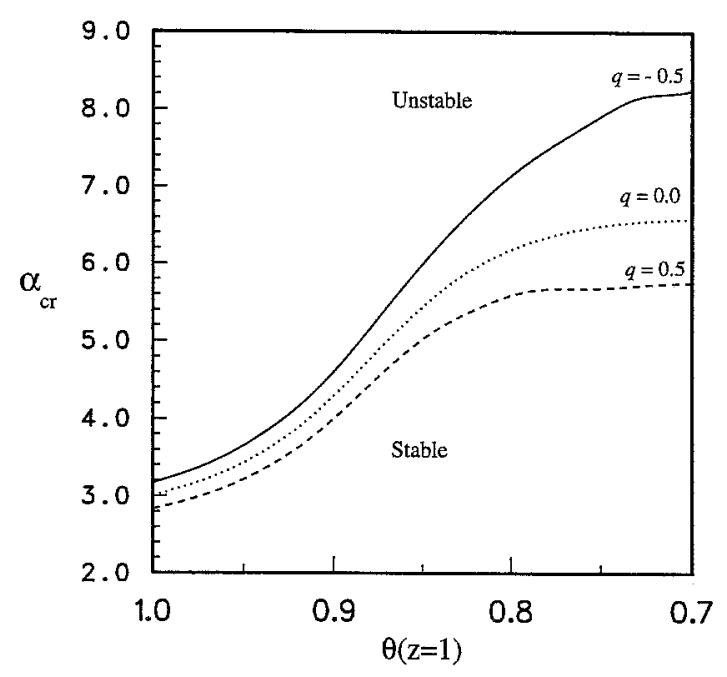

Fig. 6 Marginal stability curve for nonisothermal generalized Newtonian fiber drawing using the Walther correlation for various power-law indices, $\mathrm{Pe}=474, \mathrm{~m}=0.13$

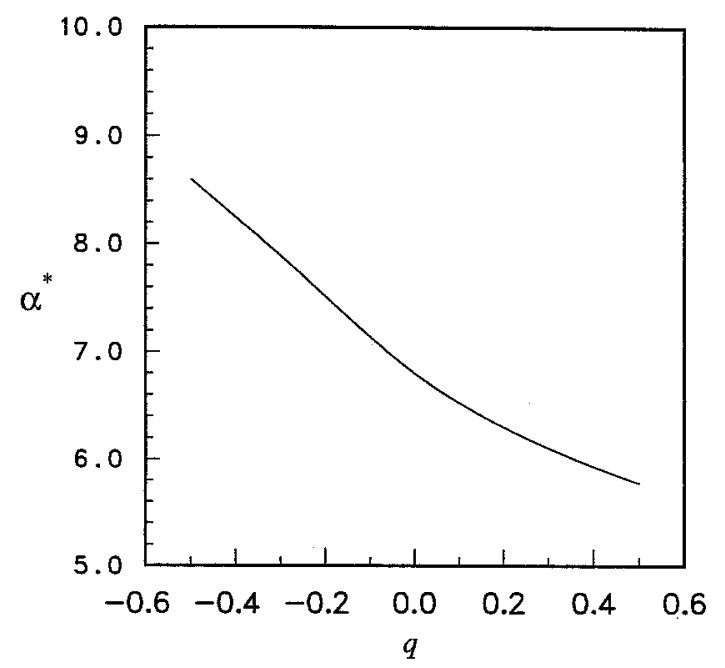

Fig. 7 Maximum critical draw ratio $\alpha *$ versus power-law index for nonisothermal generalized Newtonian fiber drawing using the Walther correlation, $\mathrm{Pe}=474, \mathrm{~m}=0.13$ 


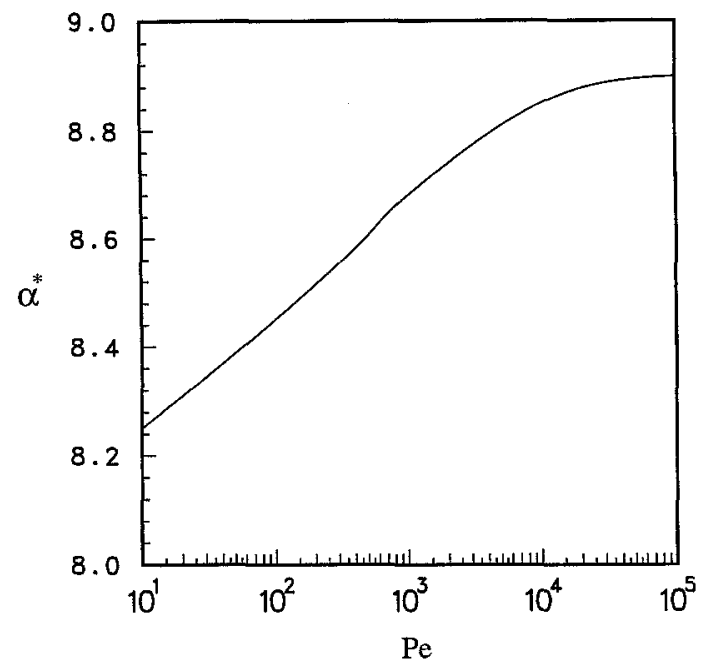

Fig. 8 Maximum critical draw ratio $\alpha *$ versus Peclet number for nonisothermal generalized Newtonian fiber drawing using the Walther correlation, $q=-0.5, \mathrm{~m}=0.13$

viscoelastic effects have been shown to have a stabilizing effect (Fisher and Denn, 1977), we examine these effects next.

\section{Viscoelastic fiber drawing}

In viscoelastic fiber drawing, as before, we find that the marginal stability curve correlates with the fiber temperature at the winder. Figure 9 shows the effect of viscoelasticity on marginal stability for an upper convected modified White-Metzner model. This figure shows that beyond a certain value of fiber temperature at the winder $\theta(z=1)$ or the heat transfer parameter $C$, we obtain unconditional stability bounded above by an unattainable region where infinite force is required to draw the fiber. The unattainable draw ratios, indicated by the region above the hatches and determined numerically where $d F / d \alpha \rightarrow \infty$, is similar to the isothermal region for the upper convected Maxwell model shown in Fisher and Denn (1976). The unattainable region indicates that the elongational viscosity becomes infinite for a finite extension rate, a failure of this simple constitutive model. The hatched region adjacent to the unattainable region indicates where the solution violates the one-dimensional assumption (Schultz, 1987). As in previous studies, we find lower and upper critical draw ratios. The upper critical curve is always near the limiting draw ratio where the one-dimensional model loses its validity. Hence, conclusions can only be drawn from the lower critical curve. A measure of the maximum draw ratio $\left(\alpha^{*}\right)$ for stable operation is determined when the critical stability curve achieves its critical value (the "nose" or "tip" as shown in Fig. 9). Since the upper part of the marginal stability curve lies close to the unat-

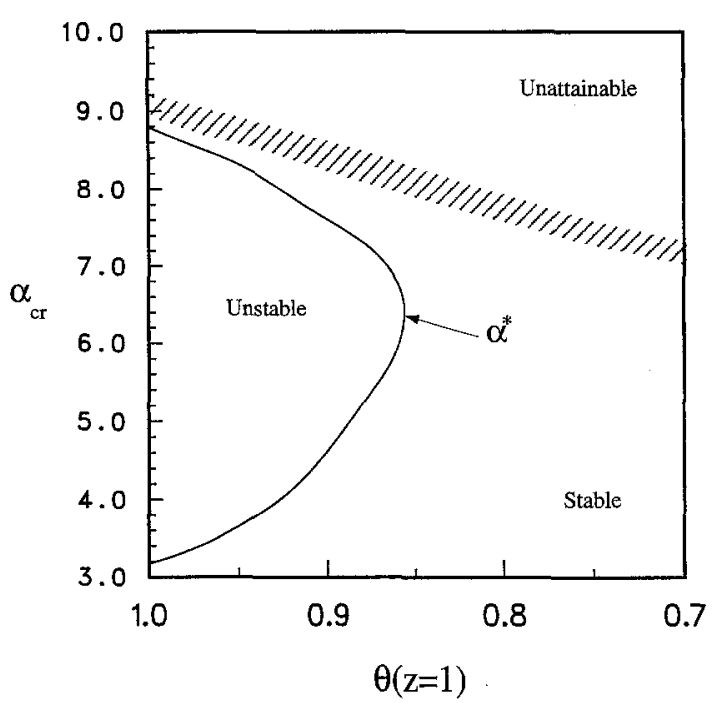

Fig. 9 Marginal stability curve for nonisothermal viscoelastic fiber drawing using the Walther correlation, $a=1, \mathrm{~m}=0.13, q=-0.5$, $\mathrm{G}=20000$

tainable region, where the one-dimensional assumption is not valid, we consider $\alpha^{*}$ as a measure of stability of the fiber drawing process. Our experience has shown that $\alpha^{*}$ is close to the unattainable region boundary as $\theta \rightarrow 0$. For this case the viscoelasticity and heat transfer have increased the draw ratio for stable operation to approximately 400 as compared to 20 for the Newtonian isothermal case. However, the draw ratio for stable operation for the generalized Newtonian nonisothermal case in the present study is $10000(q=-0.5)$ as compared to 400 for the strongly viscoelastic case $(q=-0.5)$. This indicates loss of stability when viscoelastic effects are considered.

The effect of the viscoelastic parameter $G$ on the marginal stability is shown in Fig. 10 and compares with the generalized Newtonian fiber drawing in the limit $G \rightarrow \infty$. The unattainable regions are not displayed in this figure for clarity. For all these viscoelastic cases, the lower marginal stability curve is asymptotic to the marginal curve for the inelastic fiber drawing as $\theta \rightarrow 1$. Figure 10 shows that when viscoelastic effects are combined with extensional thickening, the critical draw ratio is always lower than the inelastic limit. As viscoelastic effects are increased, the unattainable region (where $d F / d \alpha \rightarrow \infty$ ) lowers the upper critical curve. We interpret this as a loss of stability as viscoelasticity is limiting the extent the fiber can be drawn. Figure $11(\mathrm{Pe}=474 m=0.13 q=0.0)$ shows that in the absence of extensional thickening or thinning, the maximum critical draw ratio for low viscoelasticity (or higher $G$ ) is about the same as that of generalized Newtonian fiber drawing. However, as viscoelastic effects are increased, we once again observe loss of stability.

The maximum critical draw ratio $\alpha^{*}$ is shown for various viscoelastic parameters and for different power- 


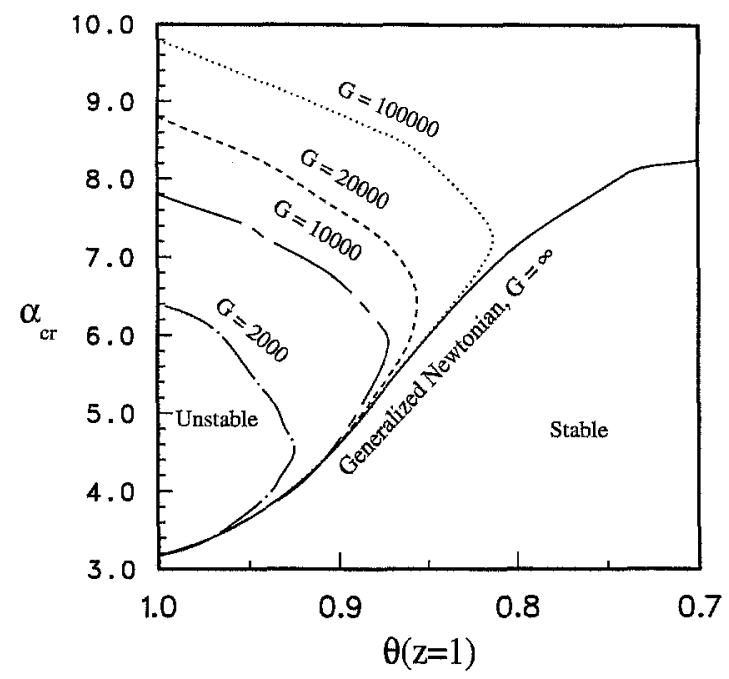

Fig. 10 Marginal stability curves for nonisothermal generalized Newtonian and viscoelastic fiber drawing using the Walther correlation, $\mathrm{Pe}=474, q=-0.5, \mathrm{~m}=0.13, a=1.0$

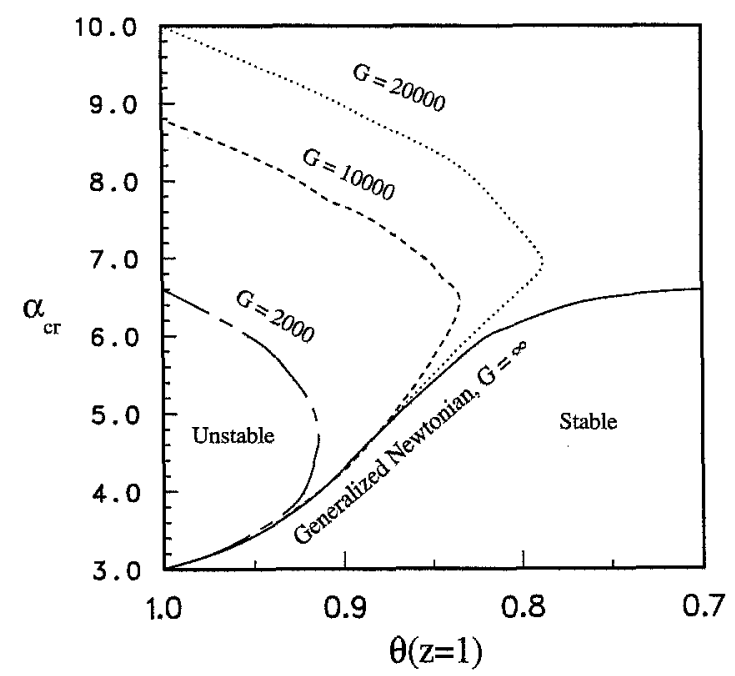

Fig. 11 Marginal stability curves for nonisothermal Newtonian and viscoelastic fiber drawing using the Walther correlation, $\mathrm{Pe}=474, q=0.0, \mathrm{~m}=0.13, a=1.0$

law indices in Figs. 12 and 13, respectively. We observe that the inelastic fiber drawing is more stable in the presence of extensional thickening effects, whereas extensional thinning helps to slightly increase the maximum critical draw ratio when viscoelastic effects are small. In contrast to generalized Newtonian fiber drawing (Fig. 7), in viscoelastic fiber drawing we do not observe (Fig. 13) an unconditional stabilizing influence of extensional thickening. We observe that there are two non-Newtonian competing factors affecting the stability of the process: viscoelasticity is generally destabilizing, extensional thickening is stabilizing. The proper balance of these two

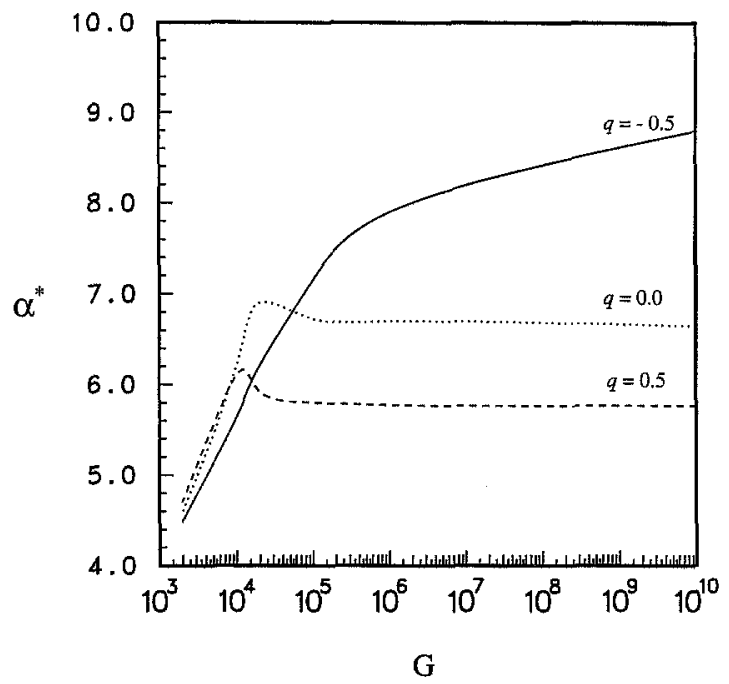

Fig. 12 Maximum critical draw ratio $\alpha *$ versus viscoelastic parameter $G$ for nonisothermal viscoelastic fiber drawing for the Walther correlation and different power-law indices, $P e=474, m=0.13$, $a=1.0$

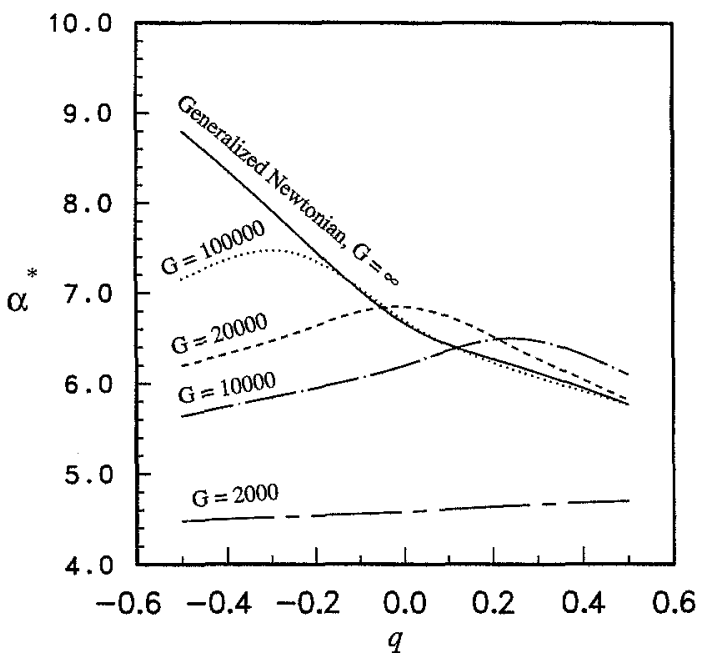

Fig. 13 Maximum critical draw ratio $\alpha^{*}$ versus power-law index for nonisothermal viscoelastic fiber drawing using the Walther correlation and different relaxation function parameters, $P e=474$, $\mathrm{m}=0.13, a=1.0$

competing factors provides the maximum stability for the viscoelastic fiber drawing. Hence, viscoelasticity in the presence of extensional thinning (or thickening) has both adverse and favorable effects on marginal stability. In general, we observe that viscoelasticity has a strong adverse effect.

In the above viscoelastic cases, we have chosen the rate parameter $a=1$, representing the upper-convected model. However, to qualitatively account for second normal stress differences, the rate parameter should be $0.4<a<0.9$ (Larson, 1988). We study the effect of $a$ on the marginal stability in Fig. 14 and observe that for a 


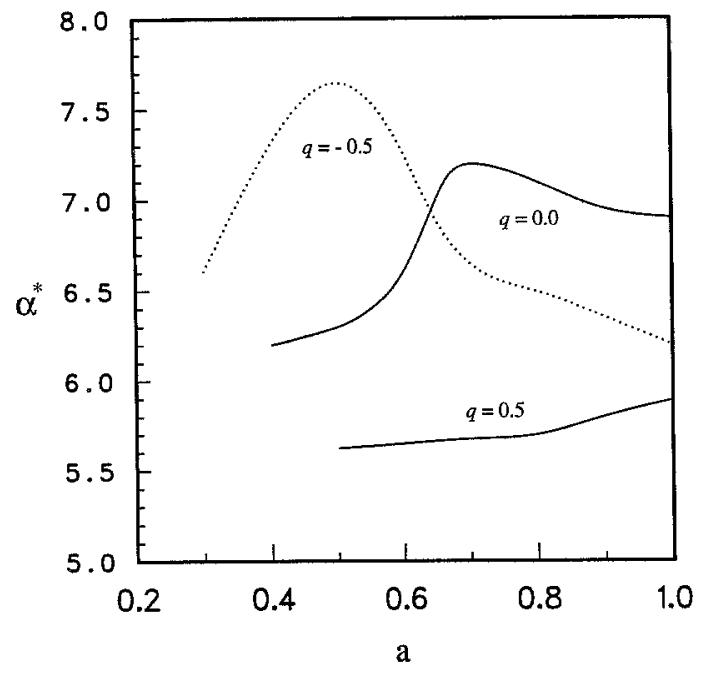

Fig. 14 Maximum critical draw ratio $\alpha *$ versus the rate parameter for nonisothermal viscoelastic fiber drawing using the Walther correlation, and different power-law indices, $\mathrm{Pe}=474, \mathrm{~m}=0.13$, $G=20000$

power-law index of $q=-0.5$ or $q=0$, a local maximum value of $\alpha^{*}$ is found for intermediate values of $a$. However, when the power-law index is 0.5 , decreasing the rate parameter results in a loss of stability, i.e., an upper-convected model $(a=1)$ predicts the maximum stability. In general, decreasing the rate parameter $a$ results in a softer material response (Larson, 1988) and hence, when extensional thickening is considered along with decreasing rate parameter $a$, there is an increase in the maximum critical draw-ratio. Once again, a proper balance of softening versus hardening due to the rate parameter, extensional thickening and viscoelastic effects determines the maximum stability. The behavior of the material when extensional thinning is taken into consideration can be explained in a similar fashion.

Finally, we analyze the effect on marginal stability when the relaxation and the viscosity temperature dependencies are different, i.e., we abandon the thermo-rheologically simple assumption and assume that the modulus $G=\eta / \lambda$ is a function of temperature, and the relaxation time $\lambda$ has the following temperature and strain-rate dependence:

$$
\lambda\left(\theta, \frac{d w}{d z}\right)=\frac{\lambda_{0}}{\left[1+\delta \frac{d w}{d z}\right]^{q}} \exp \left[\exp \left(v_{0}-v_{2} \ln \theta\right)\right],
$$

we recover thermo-rheological simplicity when $v_{1}=v_{2}$ $(G=$ constant $)$. When $v_{2}>v_{1}, G$ increases with falling temperature, and $\alpha *$ increases as shown in Fig. 15. While "small" departure from thermo-rheological simplicity rapidly improves the stability, the effects appear to asymptote to a value less than its inelastic counterpart.

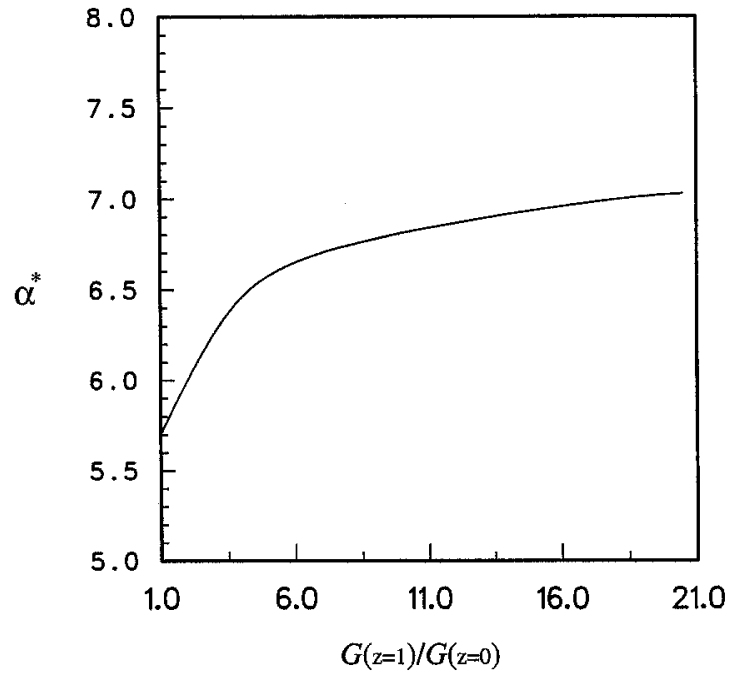

Fig. 15 Maximum critical draw ratio $\alpha^{*}$ versus the varying modulus for nonisothermal viscoelastic fiber drawing using the Walther correlation, $\mathrm{Pe}=474, \mathrm{~m}=0.13, q=-0.5, a=1, G(z=0)=10000$

\section{Concluding remarks}

We have clearly exhibited that previous numerical predictions of nonisothermal stability were underresolved. From converged eigenvalues, we have found that the nonisothermal effects stabilize the fiber-drawing operation although not unconditionally. The stability is strongly affected by the nature of the temperature-viscosity relationship. The viscoelastic model presented here has an adverse effect on process stability although extensional thickening stabilizes the fiber drawing operation.

We have found a maximum critical draw ratio to be about $10^{4}$ when heat transfer and extensional thickening are present. We also observe that the stability of the fiber is also affected by the rate parameter that accounts for the second normal stress difference. Finally, abandoning thermorheological simplicity leads to an increase in stability.

Heat transfer has increased the stable range for drawing fibers but the increase in draw ratio for stable operation is not sufficient to explain commonly attained draw ratios of $10^{5}$ in industry. We conjecture that a more complicated viscoelastic model such as a modified Jeffreys model that includes retardation effects may increase stability in modeling a hot Maxwell liquid cooling to a Kelvin solid. A more sophisticated heat transfer model is also being considered. We are currently conducting experiments and developing simulations for preform fiberdrawing draw-resonance.

Acknowledgments This study was supported by the National Science Foundation (CMS-9414891). We thank Prof. Steven Davis of Northwestern University and Dr. S. Srinivasan of Owens-Corning for sharing earlier unpublished results on this problem. 


\section{References}

Anturkar N, Co A (1988) Draw resonance in film casting of viscoelastic fluid: A linear stability analysis. J Non-Newtonian Fluid Mech 28:287-307

Bechtel SE, Forest MG, Wang Q (1992) Non-isothermal modeling of fiber spinning. ASME AMD 153/PED 141: $37-48$

Beris AN, Liu B (1988) Time-dependent fiber spinning equations. 1. Analysis of the mathematical behavior. J NonNewtonian Fluid Mech 26:341-361

Bird RB, Armstrong RC, Hassager O (1987) Dynamics of polymeric liquids, vol. 1 , 2nd ed. Wiley, New York

Cao J (1991) Studies on the mechanism of draw resonance in melt spinning. $J$ Appl Poly Sci 42:143-151

Cao J (1993) Numerical simulation of draw resonance in melt spinning of polymer fluids. J Appl Poly Sci 49:1759-1768

Chang JC, Denn MM, Geyling FT (1981) Studies on the mechanism of draw resonance in melt spinning. Ind Eng Chem Fundam 20:147-149

Chang J, Denn MM, Kase S (1982) Dynamic simulation of low-speed melt spinning. Ind Eng Chem Fundam 21:13-17

Chen Z, Papanastasiou TC (1990) Elongational viscosity by fiber spinning. Rheologica Acta 29:385-389

de Boor C (1978) A practical guide to splines. Springer, New York

Demay Y, Agassant J (1985) Experimental study of the draw resonance in fiber spinning. J Non-Newtonian Fluid Mech 18:187-198

Denn MM; Petrie CJS, Avenas P (1975) Mechanics of steady spinning of a viscoelastic liquid. AlChE J 21:791-795

Denn MM (1980) Continuous drawing of liquids to form fibers. Ann Rev Fluid Mech 12:365-387

Fisher RJ, Denn MM (1976) A theory of isothermal melt spinning and draw resonance. AIChE J 22:236-246

Fisher RJ, Denn MM (1977) Mechanics of nonisothermal polymer melt spinning. AIChE J 23:23-28

Glicksman LR (1968) The dynamics of a heated free jet of variable viscosity liquid at low Reynolds number. J Basic Engin 90:343-354
Gupta RK, Puszynski J, Sridhar T (1968) Steady spinning of the Oldroyd fluid B I: theory. J Non-Newtonian Fluid Mech 21:99-113

Gupta G, Schultz WW (1996) Nonisothermal study of Newtonian slender glass fibers. To appear in J Nonlin Mech

Kase S, Matsuo TJ (1965) Studies on melt spinning I. Fundamental equations on the dynamics of melt spinning. J Polymer Sci A 3:2541-2554

Kase S (1974) Studies on meit spinning. IV On the stability of melt spinning. J Appl Poly Sci 18:3279-3304

Larsen DC (1980) The viscoelastic response of glass during forming. Unpublished report

Larson RG (1988) Constitutive equations for polymer melts and solutions. Butterworths, Boston

Liu B, Beris AN (1988) Time-dependent fiber spinning equations. 2. Analysis of the stability of numerical approximations. J Non-Newtonian Fluid Mech 26: 363-394

Mhaskar RD, Shah YT (1977) Stability analysis of glass fibre spinning. Glass Tech 18:152-158

Myers MR (1989) A model for unsteady analysis of preform drawing. AIChE J 35:592-601

Nguyen DA, Gupta RK, Sridhar T (1990) Experimental results and constitutive modeling of the extensional flow of M1. J Non-Newtonian Fluid Mech 35: $207-214$

O'Donnell RJ, Zakarian JA (1984) Survey of two-parameter equations for the viscosity-temperature behaviour of lubricating oils. Ind Eng Chem Process Des Dev 23:491-495

Papanastasiou TC, Macosko CW, Scriven LE, Chen $Z$ (1987) Fiber spinning of viscoelastic liquid. AIChE J 33: $834-842$

Pearson JRA, Matovich MA (1969) Spinning a molten threadline: Stability. Ind Eng Chem Fund 8:605-609

Pearson JRA, Shah YT (1974) On the stability of isothermal and nonisothermal fiber spinning of power-law fluids. Ind Eng Chem Fundam 13:134-138
Pearson JRA, Shah YT, Mhaskar RD (1976) On the stability of fiber spinning of freezing liquids. End Eng Chem Fund $15: 31-36$

Petrie CJS, Denn MM (1976) Instabilities in polymer processing. AIChE J 22: 209-236

Phan-Thien N (1987) On the asymptotic behaviour of an Oldroyd fluid $B$ in steady spinning flow. J Non-Newtonian Fluid Mech 25:129-136

Sammler RL, Otaigbe JU, Lapham ML, Bradley NL, Monahan BC, Quinn CJ (1996) Melt rheology of zinc alkali phosphate glasses. J Rheol 40:285-302

Schultz WW, Davis SH (1982) One dimensional liquid fibers. J Rheol 26:331-345

Schultz WW, Davis SH (1984) Effects of boundary conditions on the stability of slender viscous fibers. J Appl Mech 106: $1-5$

Schultz WW (1987) Slender viscoelastic fiber flow. J Rheol 31:733-750

Shah YT, Pearson JRA (1972a) On the stability of nonisothermal fiber spinning. Ind Eng Chem Fundam 11: $145-149$

Shah YT, Pearson JRA (1972b) On the stability of nonisothermal fiber spinning - general case. Ind Eng Chem Fundam 11:150-153

Sridhar T, Gupta RK, Boger DV, Binnington R (1986) Steady spinning of the OIroyd Fluid B II. Experimental results. J Non-Newtonian Fluid Mech 21: $115-126$

Varshneya AK (1995) Personal communication

Vasiljev VN, Naumchic VD (1990) Analysis of the hydrodynamic stability of the glass fibre drawing process. Glass Tech 31:240-244

Wang Q, Forest MG (1994) Numerical simulations of nonisothermal fiber spinning processes. Numerical methods for non-Newtonian fluid dynamics FED-Vol 179:11-20

Zebib A (1987) Removal of spurious modes encountered in solving stability problems by spectral methods. J Comp Phys $70: 521-525$ 